\title{
Obstbau-Boom in Russland dank staatlicher Förderung, Gesundheitstrend und Umstrukturierung der Obstbauforschung
}

\author{
M. Blanke ${ }^{1}$ \\ Eingegangen: 6. Dezember 2018 / Angenommen: 9. Oktober 2020 / Online publiziert: 24. November 2020 \\ (c) Der/die Autor(en) 2020
}

\section{Zusammenfassung}

Russland kennzeichnet im Moment (2019) das schnellste Wachstum im Obstbau im Osten. Der Apfelmarkt Russlands von ca. 2,6-4,3 Mio.t Äpfeln/Jahr besteht aus 0,9-1,1 Mio.t Erwerbsobstbau, je nach Schätzung ca. 800.000t oder 2,5 Mio.t aus Hausgärten, die statistisch schwer zu erfassen sind, und ca. 1,3 Mio.t (2016), aber nur noch 0,7 Mio.t in Frostjahr 2017 Import aus Serbien, Kasachstan, Usbekistan, Tadschikistan, Aserbaidschan, Moldawien und Weißrussland sowie aus China, Chile, Neuseeland, Südafrika u. a.

Die aktuelle staatliche Förderung für Obstbau und Baumschulen beträgt - nach dem Lebensmittel-Importembargo ab August 2014 - je nach Intensität und Region von $€ 3000$ (extensiv) bis $€$ 12.000/ha (Dichtpflanzung mit Tropfbewässerung unter Hagelnetz) bzw. bis zu $80 \%$ der Anschaffungskosten für Bäume, Hagelnetze, Tropfbewässerung u. a.. Diese Anschubfinanzierung hat seit 2015 zur Neuanlage von Baumschulen, Apfelanlagen und Lagereinrichtungen geführt. Das Kapital kommt aus der Wirtschaft; die Investoren sind häufig Oligarchen. Auch in den benachbarten Regionen wie Kasachstan, Aserbaidschan und Usbekistan steigt die Obstproduktion seit 2010 stetig an.

Dieser Obstbauboom wird gestützt vom staatlichen Gesundheitstrend in Russland mit einem Ziel von $50 \mathrm{~kg}$ (heute $18-24 \mathrm{~kg}$ ) Äpfel/Kopf/Jahr - vergleichbar mit 19-23 kg (ohne Hausgärten und Streuobst) in Deutschland. Bei einem heutigen Obstkonsum (einschl. Beerenobst und Schalenobst-Nüssen) von $71 \mathrm{~kg}$ (58-64 kg ohne Schalenobst) in Russland bei einem Ziel von $106 \mathrm{~kg}$ Obst/Kopf/Jahr wäre der Selbstversorgungsgrad 22\% für Obst im Vergleich zu Deutschland mit 21,6\% (ohne Citrus, ohne Streuobst).

Eine Ausdehnung der Anbauflächen in Russland von 500.000ha (2017) auf ca. 600.000 ha (2021) am $45^{\circ} \mathrm{N}$ Breitengrad im Südwesten des größten Landes der Erde auf der Höhe der Emilia Romagna wird erwartet. Die Transportentfernungen betragen bis zu $10.000 \mathrm{~km}$. Ca. 12.000 ha sind moderne Erwerbsanlagen von Apfel auf M9 mit einem Pflanzabstand von 3,5 $\mathrm{m} \times 1 \mathrm{~m}$ unter schwarzem Hagelnetz auf heimischen Betonpfosten. Unterlage und Sortenspektrum sind mit 'Gala', 'Elstar', 'Golden Delicious', 'Granny Smith', 'Jeromine', 'Enterprise' bis 'Braeburn' auf M9 westlich geprägt - en vogue und ohne die traditionellen russischen Sorten 'Antonovka' und 'Reinette Simirenko', die weiterhin in Hausgärten angebaut werden.

Die Erzeugerpreise liegen bei $€ 0,10 / \mathrm{kg}$ für Mostobst bis fast $€ 1 / \mathrm{kg}$ für Klasse $1(65-75 \mathrm{~mm})$ für den heimischen, russischen Markt. Umstrukturierte Universitäten und Akademien der Wissenschaft konkurrieren nun um Forschungsgelder und -projekte; diese Konsolidierung resultierte 2017 in der Obstbauforschung und -züchtung in der Zusammenlegung dreier Institute zum „Michurin Federal Scientific Center“ (FNC).

Schlüsselwörter Apfel (Malus $\times$ domestica Borkh) $\cdot$ Hagelnetz $\cdot$ Antonovka $\cdot$ Kaolin $\cdot$ Klimawandel · Lebensmittel-Importembargo $\cdot$ Obstkonsum $\cdot$ Simirenko $\cdot$ Schwarzerde $\cdot$ Sonnenbrand $\cdot$ Uzbekistan

M. Blanke

mmblanke@uni-bonn.de

1 INRES-Gartenbauwissenschaft, Universität Bonn, Bonn,

Deutschland 


\title{
Expansion of State Supported Apple Cultivation, Acreage, Fruit Consumption and Restructuring of Fruit Research in Russia
}

\begin{abstract}
Russia has currently the fastest growing fruit production in the East. It's apple market of annually 2.6-4.3 milliont comprises (a) 0.9-1.1 milliont from commercial orchards, (b) 800,000 t or 2.5 million t from private gardens depending on data source, and (c) ca. 1.3 milliont (2016) but only 0.7 milliont (2017) apple imports from Serbia, Uzbekistan, Kazakhstan, Moldavia, Tadzhikistan, Azerbaijan and Belarus as well as China, Chile, New Zealand, South Africa etc.

The import embargo for EU food and state support of up to $80 \%$ of investments for new nursery trees, tree stakes, hail nets and drip irrigation led to capital investment into new orchards, nurseries and fruit stores in Russia and, similarly, in the neighbouring countries. Farm gate prices in Russia range from $€ 0.10 / \mathrm{kg}$ for industrial grade to almost $€ 1 / \mathrm{kg}$ for class $I$ apple fruit $(65-75 \mathrm{~mm})$.

These new apple orchards are planted with mostly international cvs 'Gala', 'Elstar', 'Granny Smith', 'Golden Delicious', 'Enterprise', 'Jeromine', 'Braeburn', whereas the traditional aromatic Russian varieties 'Reinette Simirenko' and 'Antonovka' remain as a specialty in private gardens. Commercial production is on a 3-wire-trellis under black hail net supported by regionally-produced concrete posts. In the favourable growing conditions of the Northern Caucasus region, the soil is often a fertile black chernosem, spring water is available from the Caucasus mountains for drip irrigation and cool nights to provide sufficient fruit colouration in the autumn, but sunburn is still problematic in orchards without hail net.

Hence, the fruit acreage is expected to expand from ca. 500,000 ha (2017) to projected ca.600,000ha by 2021 in the Southwestern corner of the world's largest country at $45^{\circ} \mathrm{N}$, the same latitude as the Emilia Romagna in Northern Italy, and sometimes necessitates fruit transport of up to $10,000 \mathrm{~km}$ to some destinations. The initiation of new orchards (ca. 12,000 ha) is in line with the Federal promotion of a healthy diet and fruit consumption with a target of $106 \mathrm{~kg}$ fruit and $50 \mathrm{~kg}$ apple/head/year, a major increase from the current $71 \mathrm{~kg}$ fruit in Russia including 18-24 kg apples/head/year.

Restructuring and streamlining of academic fruit research resulted in the amalgamation of three former institutes into the 'Michurin Federal Scientific centre (FNC)' and resulted in competition between academies of sciences and universities for grants, high publication rate and academic ranking.
\end{abstract}

Keywords Apple (Malus $\times$ domestica Borkh) $\cdot$ Chernozem $\cdot$ Climate change $\cdot$ Hail net $\cdot$ Kaolin $\cdot$ Kazakhstan Simirenko $\cdot$ Russian food embargo $\cdot$ Sunburn

\section{Einleitung}

Vielen ist Professor Budagovsky als Züchter der Apfelunterlage B9 - in USA kurz als 'Bud 9' bezeichnet - dem feuerbrand-, mehltau-, schorf- und frosttoleranten Pendant zu M9, die Apfelsorte (und Unterlage) 'Antonovka' mit der hohen Frostresistenz bis $-50^{\circ} \mathrm{C}$ sowie der Botaniker, Pflanzenzüchter und Pflanzenernährer Ivan Michurin (1855-1935) bekannt.

\section{Folgen der Konsolidierung der Obstbauforschung in Russland}

Die Obstbauforschung in Russland verteilte sich auf das nach ihm benannte Michurin All Russian Research Institute mit einem Institut in der Region Tambov, der State Universität in Michurinsk, der Landw. Fakultät an der Universität Krasnodar und dem North Caucasian Federal Scientific Center of Horticulture westlich von Sochi und der AgrarFakultät an der Kaliningrad State University in der russi- schen Enklave (Tab. 1). Ende 2017 kam es aus finanziellen Gründen und zur Effizienzsteigerung zur Zusammenlegung der drei Gartenbauwissenschaftlichen- und Züchtungsinstitute für Obst - wie das JKI in Dresden - beide befinden sich in Michurinsk und der pflanzenbaulichen Forschungsstation in der Nähe von Tambov zum neuen Michurinsk Federal Scientific Centre mit Sitz in Michurinsk (FNC 2017) mit Dr. Mikhail Akimov als Direktor, der leider kein Englisch spricht.

\section{Trendwende in der Forschung: Nun Konkurrenz um Geldmittel und Projekte}

Auch nach dem Zusammenbruch der Sowjetunion im Jahre 1991 blieben die Aufgaben getrennt - die Universitäten widmeten sich ausschließlich der Lehre, die Akademien der Wissenschaften ausschließlich der Forschung. Nun konkurrieren Universitäten und Akademien der Wissenschaft um Forschungsgelder und -projekte und ein hohes Ranking unter den russischen Universitäten (Tab. 2). 
Tab. 1 In der Obstbauforschung tätige Institute in Russland mit ihren Forschungsschwerpunkten (Stand 1. August 2018)

\begin{tabular}{|c|c|c|c|}
\hline Institution & Ort und Leiter & Obstart und Forschung & Und URL \\
\hline $\begin{array}{l}\text { Michurin All Russian Research Institute for Horticulture } \\
\text { (bis 2017) }\end{array}$ & $\begin{array}{l}\text { Michurinsk: } \\
\text { Dr Yuri Trunov }\end{array}$ & $\begin{array}{l}\text { Apfel, Pflaume, Pfirsich: } \\
\text { Anbaueignung, Frosthär- } \\
\text { te }\end{array}$ & Siehe unten \\
\hline Russian Academy of Agricultural Sciences (bis 2017) & Tambov & - & Siehe unten \\
\hline Michurinsk Federal Scientific Center (ab 2018) „FNC“ & $\begin{array}{l}\text { Michurinsk: } \\
\text { Dr. Mikhail Akimov }\end{array}$ & $\begin{array}{l}\text { Obstbau in gemäßigten } \\
\text { Breiten, Sorten, Anbau }\end{array}$ & $\begin{array}{l}\text { URL: http://fnc- } \\
\text { mich.ru// }\end{array}$ \\
\hline Michurinsk State Agricultural University & Michurinsk & Siehe oben & Siehe oben \\
\hline Lomonossow Universität - Fakultät für Biologie & $\begin{array}{l}\text { Moskau: } \\
\text { Prof. Alexei Solovchenko }\end{array}$ & $\begin{array}{l}\text { Anthozyan, Sensorik im } \\
\text { Obstbau }\end{array}$ & $\begin{array}{l}\text { URL: https://www. } \\
\text { msu.ru/en/ }\end{array}$ \\
\hline \multirow[t]{2}{*}{ Kuban Agrar-Universität Krasnodar } & $\begin{array}{l}\text { Krasnodar: } \\
\text { Prof. Tatiana Doroschenko }\end{array}$ & $\begin{array}{l}\text { Apfel: Bienenkunde, } \\
\text { Alternanz, u. a. }\end{array}$ & $\begin{array}{l}\text { URL: https:// } \\
\text { kubsau.ru/education/ } \\
\text { faculties/ }\end{array}$ \\
\hline & $\begin{array}{l}\text { Laboratory of Horticultural } \\
\text { Ecology: } \\
\text { Prof. Irina Dragavtseva }\end{array}$ & $\begin{array}{l}\text { Pektingewinnung aus } \\
\text { Kernobst }\end{array}$ & $\begin{array}{l}\text { URL: https:// } \\
\text { kubsau.ru/education/ } \\
\text { faculties/ }\end{array}$ \\
\hline $\begin{array}{l}\text { Nordkaukasisches Wissenschaftszentrum für Gartenbau, } \\
\text { Weinbau und Weinherstellung /North Caucasian Federal } \\
\text { Scientific Center of Horticulture }\end{array}$ & $\begin{array}{l}\text { Krasnodar: } \\
\text { Prof. Egorov Evgeniy } \\
\text { Alekseyevich }\end{array}$ & Obstbau & $\begin{array}{l}\text { URL: https:// } \\
\text { kubansad.ru/en/ } \\
\text { contacts/ }\end{array}$ \\
\hline $\begin{array}{l}\text { Russian Research Institute of Floriculture and Subtropical } \\
\text { Crops }\end{array}$ & $\begin{array}{l}\text { Sochi: } \\
\text { Frau Prof. Oksana Belous }\end{array}$ & $\begin{array}{l}\text { Subtropisches Obst: } \\
\text { Kaki (Persimone), Kiwi, } \\
\text { Tee u. a. }\end{array}$ & $\begin{array}{l}\text { URL: http://www. } \\
\text { vniisubtrop.ru }\end{array}$ \\
\hline Kaliningrad State; Faculty of Agronomy & $\begin{array}{l}\text { Kaliningrad: } \\
\text { Frau Prof. Elena Ronz'hina }\end{array}$ & $\begin{array}{l}\text { Source: Sink Relation- } \\
\text { ship, Apfel }\end{array}$ & $\begin{array}{l}\text { URL: http://www. } \\
\text { klgtu.ru/en/ }\end{array}$ \\
\hline
\end{tabular}

\section{Obstbauflächen in Russland}

Der Obstbau in Russland unterschiedlicher Anbauintensität konzentriert sich auf die 3 südwestlichen Provinzen (Abb. 1) auf über 500.000 ha - Ziel sind ca. 600.000 ha bis 2021 (Tab. 2). Zu 76.600 ha Erwerbsobstbau bzw. 50.000 ha Kern- und Stein-(Baum-)Obst in Deutschland (BMEL 2014) kommen nach Güll (2015) und NABU

Tab. 2 Ranking der russischen Universitäten über alle Fachgebiete. (Anonym 2018a)

\begin{tabular}{|c|c|}
\hline Forschungseinrichtung in Russland & $\begin{array}{l}\text { Zahl der Veröf- } \\
\text { fentlichungen }\end{array}$ \\
\hline (1) Lomonosov Moscow State Universität & 51.401 \\
\hline (2) Universität St. Petersburg & 4487 \\
\hline $\begin{array}{l}\text { (3) Sobolev Institut für Mathematik, Siberian } \\
\text { Branch der russ. Akademie der Wissenschaften }\end{array}$ & 3873 \\
\hline $\begin{array}{l}\text { (4) Steklov - Mathematisches Institut der russ. } \\
\text { Akademie der Wissenschaften }\end{array}$ & 3273 \\
\hline $\begin{array}{l}\text { (5) Institut für Hochtemperaturforschung der } \\
\text { russ. Akademie der Wissenschaften }\end{array}$ & 2613 \\
\hline $\begin{array}{l}\text { (6) Federal Research Center „Computer Science } \\
\text { and Control“ der russischen Akademie der Wis- } \\
\text { senschaften }\end{array}$ & 2483 \\
\hline $\begin{array}{l}\text { (7) V. A. Trapeznikov Institut der russ. Akademie } \\
\text { der Wissenschaften }\end{array}$ & 2266 \\
\hline (8) Novosibirsk State Universität & 2197 \\
\hline $\begin{array}{l}\text { (9) P. N. Lebedev Physikalisches Institut der russ. } \\
\text { Akademie der Wissenschaften }\end{array}$ & 2154 \\
\hline (10) Kazan Staatsuniversität & 2112 \\
\hline
\end{tabular}

ca. 200.000 ha Streuobst mit abnehmender Tendenz - der größte Teil davon Apfel; für Hausgärten in Deutschland entfallen bei den Kleinstparzellen Angaben über Hektarzahlen.

\section{Obstkonsum in Russland - bekannte Sorten - neue Lieferanten}

Der Apfelmarkt Russlands von 2,6-4,3 Mio.t Äpfel jährlich teilt sich auf in 1,1-1,7 Mio.t aus Erwerbsobstbau, 2,3-2,6 Mio.t aus Hausgärten, die statistisch nicht genau erfasst werden können und ca. 1,3 Mio.t Importe (2016), aber nur noch ca. 0,7 Mio.t Importe im Frostjahr 2017 (Abb. 2).

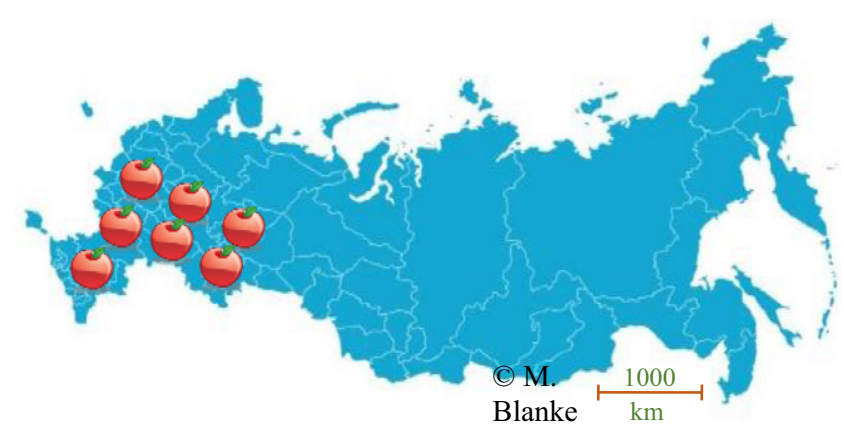

Abb. 1 Lage der Obstanbaugebiete im äußersten Südwesten des größten Landes der Erde 


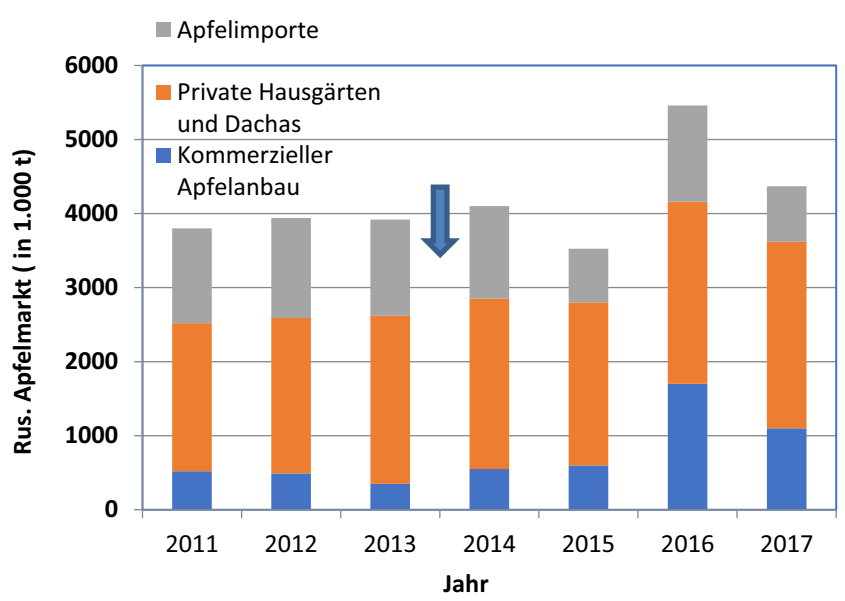

Abb. 2 Der russische Apfelmarkt nach Anbaumenge (der Pfeil markiert das Inkrafttreten des Lebensmittel-Importembargos im August 2014)

Ursprungsländer der Äpfel sind heute neben Serbien (Blanke 2020), Usbekistan, Moldawien, Tadschikistan, Aserbaidschan, Weißrussland (Belarus) und China, Chile (Blanke \& Yuri 2020), Neuseeland und Südafrika u.a. sowie Kasachstan, wo sich die Erntemenge von ca. $100.000 \mathrm{t}$ auf ca. fast 200.000t verdoppelte (ca. 32.000 ha; Abb. 3). In Aserbaidschan gibt es seit 2012/3 Entrepreneurship Anschubfinanzierungen für den Agrarsektor und die Erntemenge (Kern- und Steinobst einschl. Beeren) verdoppelte sich von ca. 0,5 Mio.t in 2004 auf fast 1 Mio.t Obst heute - mit Exportziel Russland - parallel zu einer Flächenausdehnung von 90.000 ha (2005) auf 190.000 ha Obstbau heute (Anonym 2018b); ähnlich verdoppelte sich die Apfelernte in Usbekistan von 500.000t in 2007 auf heute über 1,2 Mio.t auf 100.000 ha (FAO 2018) (Abb. 4).

\section{Obst- und Apfelkonsum in Russland}

Der Obstverzehr in Russland beträgt ca. $71 \mathrm{~kg} / \mathrm{Kopf} /$ Jahr einschl. Beerenobst und Schalenobst (Nüssen) bzw. 58-64 kg ohne Schalenobst (Tab. 3) (Rosstat 2017). Ziel der russischen Gesundheitskampagne (Verordnung N 614 des Gesundheitsministeriums vom 15. August 2016 [Ano-

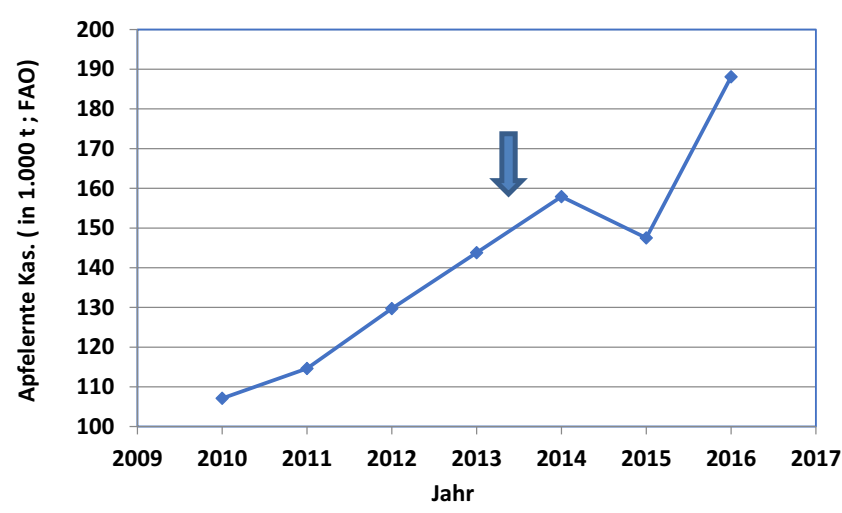

Abb. 3 Entwicklung der Apfelerntemengen in Kasachstan (Anonym 2018a; nach FAO und http://stat.gov.kz) (der Pfeil kennzeichnet den Beginn des Importembargos für frische Lebensmittel aus Westeuropa nach Russland)

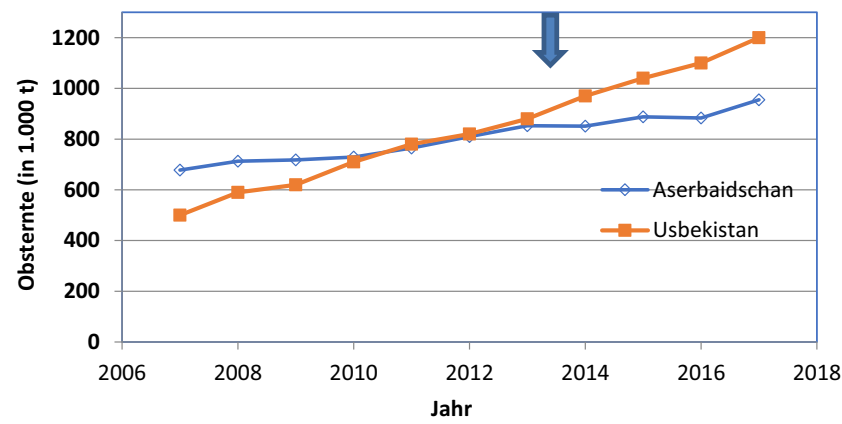

Abb. 4 Entwicklung der Obsterntemengen (einschl. Beerenobst) in Aserbaidschan (Anonym 2018b; nach http://www.stat.gov.az/source/ agriculture/) und Usbekistan (FAO 2018) (der Pfeil kennzeichnet den Beginn des Importembargos in Russland, der den Anstieg des Obstbaus beschleunigte)

nym 2017]) ist ein Apfelkonsum von $50 \mathrm{~kg} / \mathrm{Kopf} / \mathrm{Jahr}$. Im Vergleich dazu liegt der Obstkonsum in Deutschland bei $119 \mathrm{~kg} / \mathrm{Kopf} / \mathrm{Jahr}$, wenn man zur Versorgungsbilanz Obst von $67,4 \mathrm{~kg} / \mathrm{Kopf} / \mathrm{Jahr}$ (Garming et al. 2018) und 34,2 kg/ Kopf/Jahr für Citrusfrüchte (BMEL 2014) geschätzte $50 \%$, d.h. $12 \mathrm{~kg}$ Frischware aus den geschätzten $25 \mathrm{~kg} / \mathrm{Kopf} /$ Jahr aus Hausgärten und Streuobst und die $4 \mathrm{~kg}$ für Nüsse (BMEL 2014) hinzurechnet (Tab. 3).

Tab. 3 Vergleich des Pro Kopf-Frischobst- und Apfelkonsums in Russland und Deutschland (jeweils einschl. Hausgärten, Citrus und Schalenobst/ Nüssen)

\begin{tabular}{|c|c|c|c|c|}
\hline & \multicolumn{2}{|c|}{ Russland } & \multicolumn{2}{|c|}{ Deutschland } \\
\hline & Obst & Tafeläpfel & $\overline{\text { Obst }}$ & Tafeläpfel \\
\hline Obstkonsum [Mio.t] & 10,3 & $3-4$ & 9,8 & 1,5 \\
\hline Ziel in Russland [kg/Kopf/Jahr] & 106 & 50 & - & - \\
\hline Konsum Frischobst $[\mathrm{kg} / \mathrm{Kopf} / \mathrm{Jahr}]$ & $71^{\mathrm{a}}$ & 24 & $119^{\mathrm{a}}$ & $25^{\mathrm{b}}$ \\
\hline Selbstversorgungsgrad (SVG) Frischobst [\%] & $22,1^{\mathrm{a}}$ & Ca. 65 & 20 & Ca. 43 \\
\hline
\end{tabular}

${ }^{a}$ mit Hausgärten einschl. Beerenobst und Nüssen

${ }^{\mathrm{b}}$ ohne Hausgärten und ohne Streuobst 


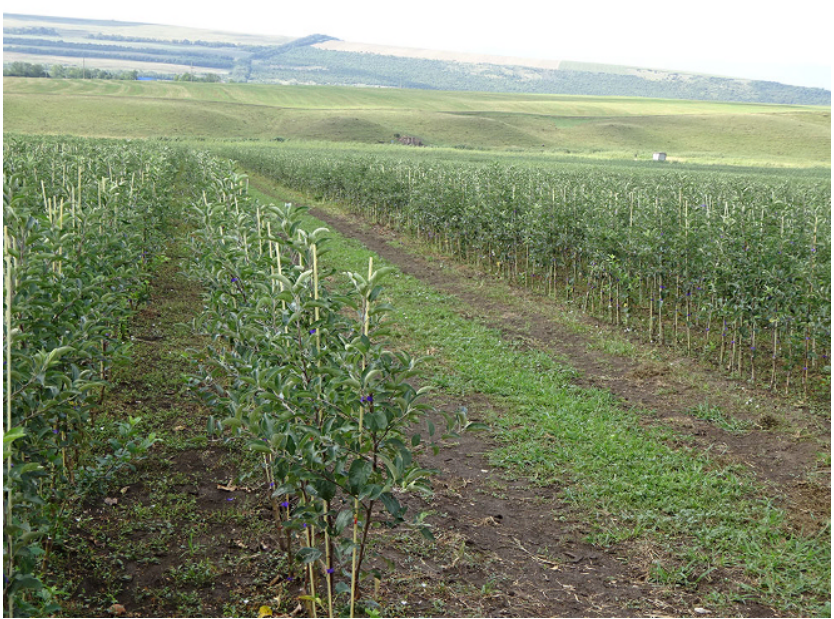

Abb. 5 In 2015 neu gegründete Baumschule Stavropol'ya in 2018

\section{Herkunft der Apfelimporte}

Das Lebensmittel-Importembargo Russlands seit August 2014 trifft frische Lebensmittelimporte aus Europa, Norwegen, USA, Kanada und Australien und zeit- (März 2016-März 2018) bzw. teilweise der Türkei. Ausgenommen ist Zuchtmaterial tierischer und pflanzlicher Herkunft, d.h. Pflanzmaterial und Zubehör wie Hagelnetze, Beregnung, Lagertechnik, etc.. Das Importembargo stärkte nicht nur den Obstbau im eigenen Land und Handel mit heimischen Produkten, sondern auch der Nachbarländer Moldawien und Kasachstan (Abb. 3) sowie Usbekistan und Aserbaidschan (Abb. 4). Wenn Serbien Mitglied der EU wird, entfällt seine Exportmöglichkeit nach Russland (Blanke 2020). Durch das angespannte Verhältnis zur Ukraine,

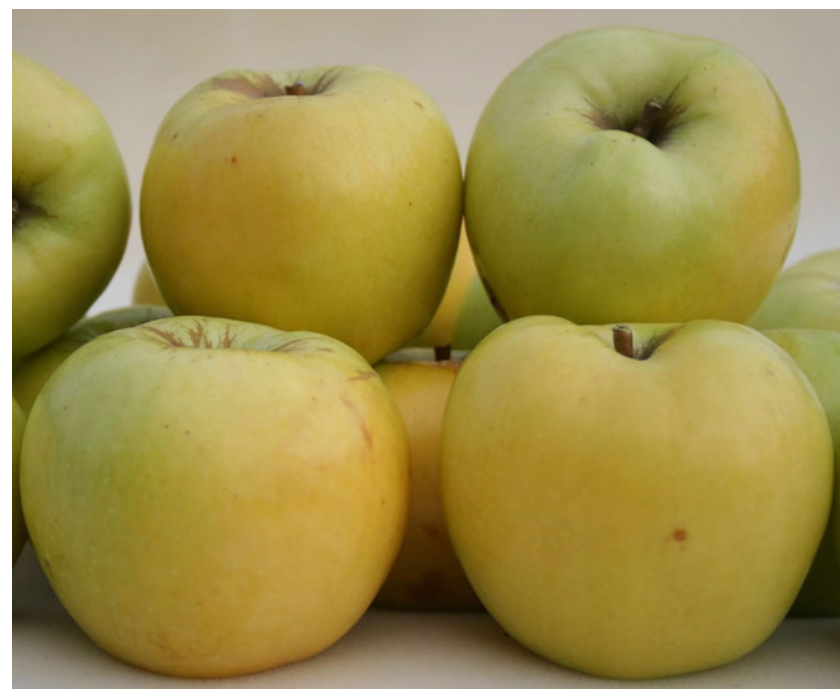

Abb. 7 'Antonovka', alte aromatische russische frostharte Apfelsorte. (Aus https://yagodka.club/frukty/’ Antonovka'-sort-yablok.html)

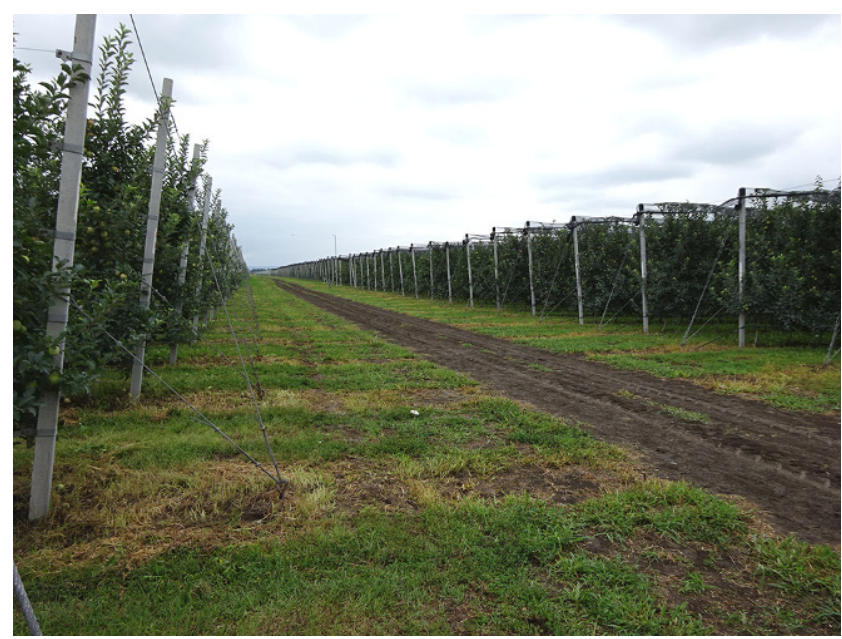

Abb. 6 Neue Apfelanlagen unter schwarzem Hagelnetz bei Sad Gigant Inguschetien in 2018

exportiert das Land - trotz der günstigen Lage - seine steigenden Apfelmengen nach Europa und Übersee.

\section{Staatliche Förderung für Neuanlagen und Investitionen für den Obstbau und Baumschulen}

Die aktuelle staatliche Förderung von Neuanlagen reicht von $€ 700 /$ ha für Beerenobstbetriebe (Freiland, ohne Überdachung), $€ 3175 /$ ha für Obstbaumschulen (Abb. 5) und $€$ 3000/ha (extensive Anlagen) bis zu maximal $€ 12.000 /$ ha für Dichtpflanzungen mit Tropfbewässerung unter Hagelnetz (Abb. 6; Tab. 4), wobei die Auszahlungssummen von Jahr zu Jahr schwanken können. Die Anschubfinanzierung

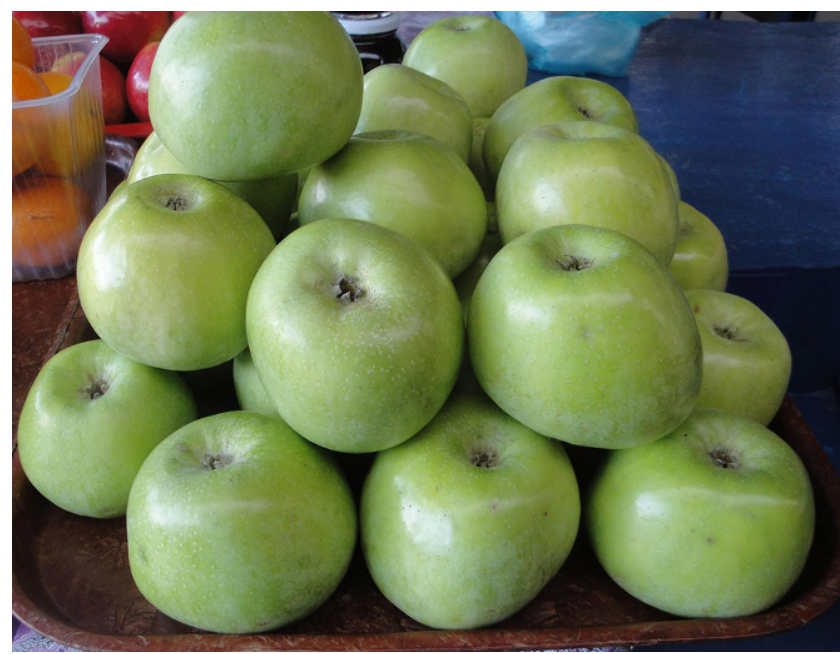

Abb. 8 'Simirenko', alte traditionelle, beim russischen Konsumenten wegen seines Aromas sehr geschätzte Sorte 
Tab. 4 Größenordnungen der aktuellen staatlichen Anschubfinanzierung im Obstbau und Baumschule pro ha-Werte schwanken je nach Anbauregion, Pflanzdichte und Jahr und Haushaltslage

\begin{tabular}{ll}
\hline Betrieb & Staatliche Förderung (in $€ / h a)$ \\
\hline Baumschulen & Ca. 3175 \\
Baumobst & Ca. $3600-12.000$ \\
Beerenobst & Ca. 700 \\
Obergrenze pro Betrieb & - \\
\hline
\end{tabular}

von ca. $€ 3000 /$ ha für die Neuanlage von Baumschulen (Abb. 5) in Russland ist in ähnlicher Größenordnung wie die für Neuanlagen einfacher Obstanlagen ohne Hagelnetze und ohne Tropfbewässerung; ähnliche Förderungen gibt es auch für den Gemüsebau.

In der im Rahmen von RuGeFren im August 2018 besichtigten Baumschule Sadi Stavropol'ya (Abb. 5) wurden die drei Apfelsorten 'Granny Smith' und 'Golden Delicious' und 'Fuji' auf M9 vermehrt und das westlich bzw. international ausgerichtete Apfelsortiment gezielt für den russ. Markt und die dort en vogue Sorten bestimmt. Die virusgetesteten Apfelbäume hatten EU-Exportqualität, wenn auch noch ohne Zertifizierung; bei den russ. Baumschulen gibt es auch westliche Baumschulen als Investoren. Neben der Vermehrung verfügte die besichtige Baumschule auch über Labors für Virustestung und Mineralstoffanalysen sowohl von Blättern, Früchten als auch Böden, da die Nährstoffgehalte der Böden in der Region Stavropol stark schwanken können.

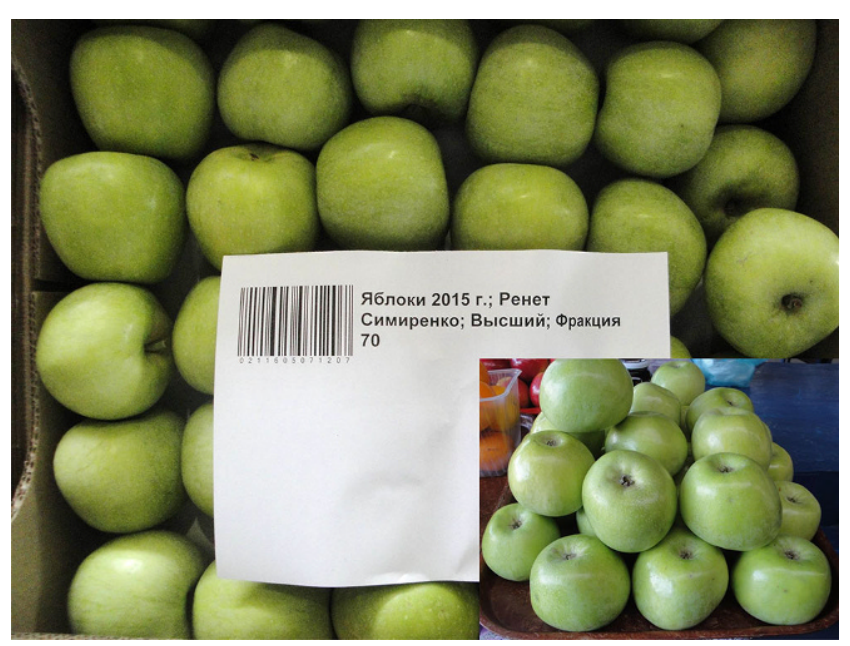

Abb. 9 Traditionelle russische Apfelsorte 'Simirenko'
Tab. 5 Erzeuger- und Supermarktpreis für Äpfel in 2018 (ca. 80 Rubel $-€ 1)$

\begin{tabular}{llllll}
\hline Handelsklasse & \multicolumn{2}{l}{ Erzeugerpreis/kg } & & \multicolumn{2}{l}{ Supermarktpreis/kg } \\
\cline { 2 - 3 } & $\begin{array}{l}\text { In } \\
\text { Rubel }\end{array}$ & $\begin{array}{l}\text { In Euro- } \\
\text { cent }\end{array}$ & $\begin{array}{l}\text { In Ru- } \\
\text { bel }\end{array}$ & In Euro \\
\hline $\begin{array}{l}\text { Klasse } 1 \\
(65-75 \mathrm{~mm})\end{array}$ & $65-70$ & $81-88$ & 180 & 2,20 \\
$\begin{array}{l}\text { Klasse } 2(\text { leichte } \\
\text { Schäden) }\end{array}$ & 40 & 50 & 120 & 1,50 \\
Mostobst & $7-8$ & 10 & n.v. & \\
\hline
\end{tabular}

n. v. nicht vertreten

\section{'Antonovka' und 'Simirenko' als traditionelle aromatische russische Äpfel und Unterlage B9 kaum im Erwerbsanbau}

Die großfruchtigen grünen Sorten 'Antonovka' (Abb. 7) und 'Reinette Simirenko' (Abb. 8 und 9) sind beliebte, traditionelle aromatische alte russische Apfelsorten und erklären die dortige Beliebtheit unserer übergroßen 'Golden Delicious“ - Fruchtexporte vor dem Embargo. Die Sorte geht auf den Pomologen Leo Simirenko in der Ukraine zurück, der sie 1895 in seinem Garten entdeckt haben soll. Vergleicht man den grünen 'Simirenko' der gleichen Fruchtfarbe wegen mit 'Granny Smith', ist 'Simirenko' weniger säuerlich, aromatischer und knackiger, aber sonst von vergleichbarer guter Fruchtfleischqualität und wurde schon mit zu den russischen Kosmonauten ins Weltall gesandt (Blanke 2014); die Sorte soll weitgehend identisch mit 'Woods Greening ' in Nordamerika sein. Der Anbau der Apfelsorte 'Simirenko' ist schwierig - die Sorte ist anfällig für Stippe und Glasigkeit und bringt geringe Erträge.

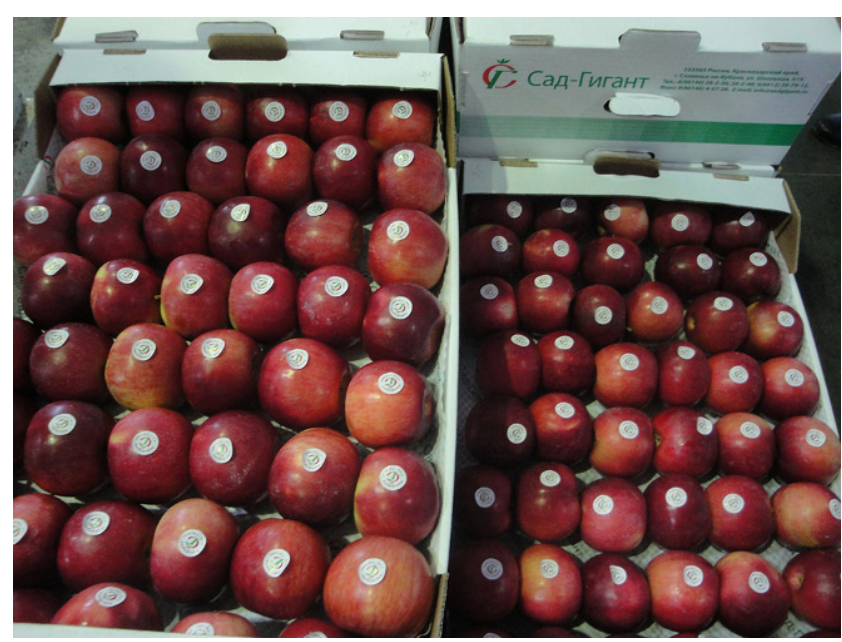

Abb. 10 'Idared' gelabelt, Klasse I bei Sad Gigant aus der Kubanregion 
Tab. 6 Anbauflächen in Russland und Deutschland (Rosstat 2017; BMEL 2014)

\begin{tabular}{llll}
\hline Obstart & $\begin{array}{l}\text { Russland } \\
{[\text { ha] }}\end{array}$ & $\begin{array}{l}\text { Deutschland } \\
{[\text { ha] }}\end{array}$ & Obstart \\
\hline Obstanlagen & $\mathbf{5 1 0 . 0 0 0}$ & $\mathbf{2 7 6 . 6 0 0}$ & Obstanlagen \\
Davon Obst in Hausgärten & 309.000 & 200.000 & Davon Streuobst in D \\
& & 76.600 & Davon Erwerbsobstbau D \\
Davon Apfelanlagen & 160.000 & 50.000 & Davon Baumobst in D \\
Davon intensive Anlagen & 12.000 & $34.000^{\mathrm{a}}$ & Davon Apfelanlagen in D \\
Obst in Planung bis 2021 & $\mathbf{7 8 . 0 0 0}$ & 23.100 & Davon Beerenobst in D \\
\hline
\end{tabular}

aohne Hausgärten

\section{Staatliche Förderung für Neuanlagen führen zu kostendeckenden Erzeugerpreisen}

Die Kombination aus staatlicher Förderung von Neuanlagen und das Import-Embargo (Tab. 4) unterstützt nicht nur die Anlage neuer Baumschulen, Apfelanlagen und Lagereinrichtungen, sondern erhöhte - nach dem Wegfall der Schutzzölle durch Russlands Beitritt zur WTO im Jahre 2012 - auch die Absatz- und Supermarktpreise für Äpfel aus heimischem russischem Anbau, aber auch ihre Wertschätzung (Tab. 5). Damit werden die Erzeuger in die Lage versetzt, rentabel Obst anzubauen. Die Einkaufs- bzw. Erzeugerpreise von Supermarktketten reichen von umgerechnet $€ 0,10 / \mathrm{kg}$ für Mostobst (Tab. 5) bis fast $€ 1 / \mathrm{kg}$ für Klasse 1 (65-75 mm, einzeln gelabelt, 'Idared') (Abb. 10).

\section{Kapital aus der Wirtschaft sichert die Versorgung des Landes}

Manche Investoren sind eher auf Gewinn als auf Nachhaltigkeit ausgerichtete Oligarchen - auch z. B. aus den USA für Lagereinrichtungen; kleine gewachsene Familienbetriebe haben kaum Zugang zu Krediten und organisieren dafür Straßenverkauf an strategisch günstigen Stellen oder liefern an Wochenmärkte. Die großen Betriebe bringen vor allem zur Erntezeit die Notwendigkeit der Bewachung gegen Diebstahl mit Securitykräften mit sich. Absatzmärkte dieser großen Mengen Obst sind die großen Supermarktketten Russlands (Tab. 6), angeführt von dem Supermarkt „X5“ oder auch 'Magnit'. Aber auch deutsche Supermarktketten wie Globus (,Real') setzten in 2017 mit ihrer z.Zt. in Russland konkurrenzlosen Strategie der ,produzierenden Händler" und großer Supermärkte 1,43 Mrd. $€$ in Russland um mit guter Lieferzufriedenheit russischer Ware und Lieferanten und 5\% Umsatzplus pro Jahr.

\section{Geographische Lage und Anbaubedingungen - fruchtbare Böden, Zugang zu sauberem Wasser und günstiges Mikroklima}

Die Anbaugebiete im größten Land der Erde am $45^{\circ} \mathrm{N}$ auf der Höhe der Emilia Romagna liegen im äußersten Südwesten (Abb. 1); von dort muss das ganze Land über weite Strecken - bis zu $10.000 \mathrm{~km}$ nach Wladiwostok - per Flugzeug, LKW und Schiene versorgt werden. Fruchtbare Schwarzerde (Tschernozem) mit pH 7,0 und Zugang zu frischem Quellwasser aus den Bergen des Kaukasusgebirges im Süden sind phantastische Voraussetzungen für Obstbau im Nordkaukasus; schwierig kann der hohe Humusgehalt werden, der in Extremfällen bis zu $8 \%$ beträgt, und der Salzgehalt durch das ehemalige Meer mit bis zu $2 \%$.

\section{Das Phänomen Schwarzerde (Tschernozem)}

Der Obstbau erfolgt in der Kubanregion auf schwierigen sowie im Nordkaukasus auf fruchtbaren Böden. Der Name Tschernozem leitet sich aus dem Russischen (Chernozjom $=$ Schwarzerde) ab und deutet auf einen dunkel gefärbten, humosen Boden. Voraussetzung für die Entstehung von Schwarzerde ist kalkhaltiges Ausgangssubstrat, z.B. Löss,

Tab. 7 Voraussetzungen zur Entstehung von Schwarzerde (Chernozem)

\begin{tabular}{lll}
\hline Ausgang & Mechanismus & Wirkung \\
\hline $\begin{array}{l}\text { Kalkreiches Ausgangsge- } \\
\text { stein }\end{array}$ & Erhält neutralen, leicht alkalischen $\mathrm{pH}$ & $\begin{array}{l}\mathrm{pH} \text { bleibt um pH 7, fördert Mikrobenaktivität und Nährstoffverfüg- } \\
\text { barkeit }\end{array}$ \\
$\begin{array}{l}\text { Leicht zersetzbare Streu } \\
\text { Bodenmikroben }\end{array}$ & $\begin{array}{l}\text { Eiweißreich } \\
\text { Bodendurchmischung }\end{array}$ & $\begin{array}{l}\text { Verlagerung organischer Substanz in den Unterboden } \\
\text { Trockener heißer Sommer }\end{array}$ \\
Langer kalter Winter & $\begin{array}{l}\text { Kurzer Herbst } \\
\text { Kurzes Frühjahr }\end{array}$ & organische Substanz nachhaltig langfristig an
\end{tabular}




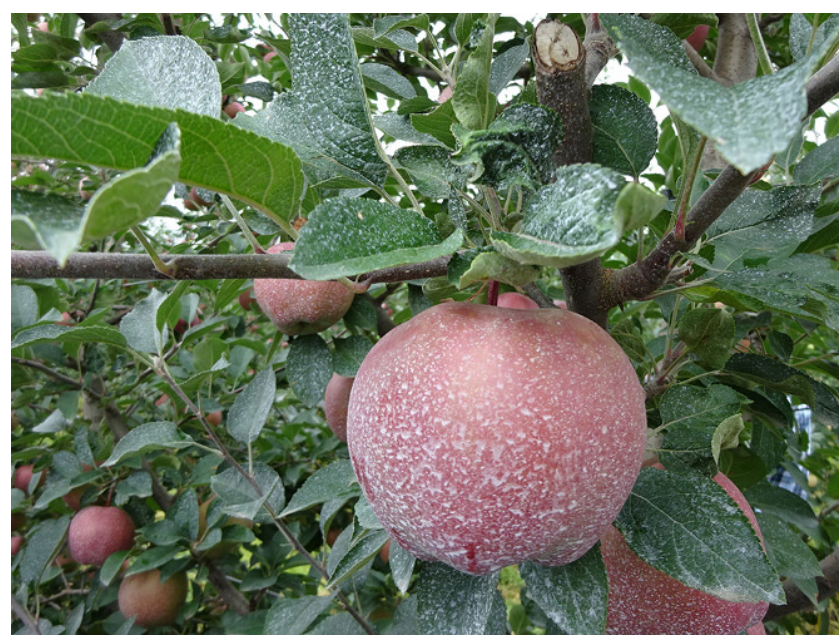

Abb. 11 Kaolin auf der Apfelsorte 'Jeromine" ohne Hagelnetz als Schutz gegen Sonnenbrand

wie aus dem Kalksandstein des Kaukasusgebirges. Es erhält einen neutralen $\mathrm{pH}$-Wert, gute Mikrobenaktivität und gute Nährstoffversorgung - vor allem im Obstbau auch mit Calcium - ohne Nährstoff-Festlegung. Die zweite Voraussetzung ist kontinentales Klima mit kurzem Herbst, kurzem Frühjahr und langem Winter, aber auch mit heißem, trockenen Sommer, in denen die humose Streu mineralisiert wird und die Anreicherung organischer Substanz (Humus) im Oberboden (Tab. 7) ermöglicht.
Als dritte Voraussetzung sorgen Bodenorganismen für eine Durchmischung des Bodens und Verfrachtung organischer Substanz in tiefere Schichten mit Krotowinen-Bildung, den hellen und dunklen Flecken am Übergang vom humosen zum mineralischen Boden; dies sind ehemalige neu verfüllte Grabgänge.

Die Fruchtbarkeit der Schwarzerde kann sich erst entfalten, wenn in den trockenen heißen Sommern Wasser zur Verfügung steht. Andererseits kann Schwarzerde durch übermäßige Bewässerung degradieren, die Lebensbedingungen für die Mikroorganismen werden zwar verbessert, aber die organische Substanz wird schneller mineralisiert. Außerdem können Tonverlagerungen die Bodenstruktur verschlechtern und zu einem anderen Bodentyp führen.

\section{Anpassungsstrategien an die Klimawandelfolgen im Nordkaukasus}

Im Nordkaukasus färben die Herbstsorten durch die kühle Gebirgsluft des Kaukasus; gegen Sonnenbrand werden vor allem in Anlagen noch ohne Hagelnetz - erst $2 \times$ Kaolin (Abb. 11) und dann $2 \times$ UV-Filter (purshade) erfolgreich eingesetzt. Fast alle neuen Anlagen werden mit schwarzem Hagelnetz ausgestattet; als Pfosten dienen Betonpfähle aus heimischer Produktion.

Aktueller Schwerpunkt des Forschungsaustausches und der wissenschaftlichen Kooperation RuGeFren (https://

Tab. 8 Anpassungsstrategien auf Klimawandelfolgen für den Obstbau im Nordkaukasus

\begin{tabular}{|c|c|c|c|}
\hline Wetterphänomen & Wirkung & Wetterphänomen & Adaptionsstrategien \\
\hline Mehr Hitzetage & $\begin{array}{l}\text { Verringerte Photosynthese, längere Fruchtentwicklung, } \\
\text { kleinere Früchte }\end{array}$ & Warmer Herbst & $\begin{array}{l}\text { Ausfärbung bei Herbstsorten durch kühle } \\
\text { Gebirgsluft gut }\end{array}$ \\
\hline $\begin{array}{l}\text { Warmer Som- } \\
\text { mer }\end{array}$ & Knospeninduktion 3 Wochen verzögert & $\begin{array}{l}\text { Warmer Som- } \\
\text { mer }\end{array}$ & Schlechte Ausfärbung bei Sommersorten \\
\hline Herbstfrost & Knospen erfrieren & Sonnenbrand & Kaolin + UV (Abb. 10) \\
\hline $\begin{array}{l}\text { Wärmerer } \mathrm{Au}- \\
\text { gust }\end{array}$ & Später Eintritt in die Dormanz & Hagel & Schwarze Hagelnetze \\
\hline $\begin{array}{l}\text { Wärmere Win- } \\
\text { ter }\end{array}$ & Unzureichende Erfüllung des Kältebedürfnis & Frost selten & Kein Frostschutz \\
\hline
\end{tabular}

Tab. 9 Trendwenden im russischen Obstbau und in der Obstbauforschung

\begin{tabular}{|c|c|c|}
\hline Parameter & Vor dem Import-Embargo & Nach dem Embargo \\
\hline Obstbau-Forschung & $\begin{array}{l}\text { Forschung bisher nur an den russischen Aka- } \\
\text { demien der Wissenschaft }\end{array}$ & Forschung nun auch neben Lehre an den Universitäten \\
\hline Ernährung/Gesundheitstrend & Geringe Ernährung bzw. Konsum von Obst & Ziele: $106 \mathrm{~kg} / \mathrm{Obst} / \mathrm{Kopf} 50 \mathrm{~kg}$ Äpfel/Kopf $/ \mathrm{Jahr}$ \\
\hline Anschub-Finanzierung & Keine & Siehe Tab. 4 \\
\hline Nachhaltigkeit & $\begin{array}{l}\text { Kaum Hagelnetze und kaum Bewässerung, } \\
\text { kaum Schutz vor Sonnenbrand }\end{array}$ & Hagelnetze und Bewässerung sind Standard in Neuanlagen \\
\hline Neuanlagen & $\begin{array}{l}\text { Kaum Neuanlagen - uninteressant für Inves- } \\
\text { toren }\end{array}$ & $\begin{array}{l}\text { Neue Obstanlagen und neue Baumschulen - attraktiv für } \\
\text { Investoren aus dem In- und Ausland }\end{array}$ \\
\hline $\begin{array}{l}\text { Wertschätzung für Obst russi- } \\
\text { scher Herkunft }\end{array}$ & Gering & Gut \\
\hline
\end{tabular}


www.rugefren.wordpress.com) sind die gemeinsame Entwicklung und Austausch von Anpassungsstrategien an die Klimawandelfolgen für den Obstbau in beiden Ländern (Tab. 8).

Im Nordkaukasus ist ein Ziel beim Apfelanbau eine Ertragssteigerung in den modernen Anlagen (ca. 12.000 ha) von heute ca. 60 auf $78 \mathrm{t} / \mathrm{ha}$ bei Apfel. Neben dem Anbau von Äpfeln erlaubt das Klima dort den Anbau von Pfirsichen, Kirschen, Nektarinen, Pflaumen und Aprikosen und in Kürze - Tafeltrauben, da die Weinbereitung durch die muslimische Religion in Inguschetien nicht möglich ist.

\section{Fazit}

Die Kombination aus Import-Embargo, staatl. verordneten Gesundheitstrend und staatl. Förderhilfen für Investitionen haben den Obstbau in Russland - wie beabsichtigt - gestärkt, zur Neuanlage vieler Baumschulen und Obstanlagen geführt und den heimischen Obstbau finanziell rentabel gemacht (Tab. 9) und sich auch positiv auf die Obstproduktion der Nachbarländer wie Kasachstan ausgewirkt. Auch in naher Zukunft werden noch Importe notwendig sein, um den Obstkonsum zu decken und die ehrgeizigen Ziele des Gesundheitstrends zu erreichen. Die Angebotsbreite an Obst blieb erhalten, aber mit anderer Herkunft bei etwas gestiegenen Preisen vor allem in den Supermärkten - bei stabilem Rubelwechselkurs. Gesundheitstrend und Nachfrage nach regionalen Produkten haben die Erzeugerpreise und den Optimismus der Obstbauern bzw. Investoren begünstigt, die vorher in Konkurrenz zu europäischen Billigimporten bei Früchten und Bäumen standen.

Danksagung Ich danke Dr. Anton Milyaev, Universität Hohenheim für die Hilfestellung bei den Übersetzungen und Interpretationen sowie die kritische Durchsicht des Textes, Dr. Alexey Solomakhin und vielen russ. Kollegen für Unterstützung bei der Suche nach den Statistiken, Dr. Dereck P Hucklesby, Bristol, UK für die Durchsicht des englischen Abstracts und dem BMEL für die Unterstützung dieser wissenschaftlichen Zusammenarbeit.

Funding Open Access funding enabled and organized by Projekt DEAL.

Interessenkonflikt M. Blanke gibt an, dass kein Interessenkonflikt besteht.
Open Access Dieser Artikel wird unter der Creative Commons Namensnennung 4.0 International Lizenz veröffentlicht, welche die Nutzung, Vervielfältigung, Bearbeitung, Verbreitung und Wiedergabe in jeglichem Medium und Format erlaubt, sofern Sie den/die ursprünglichen Autor(en) und die Quelle ordnungsgemäß nennen, einen Link zur Creative Commons Lizenz beifügen und angeben, ob Änderungen vorgenommen wurden.

Die in diesem Artikel enthaltenen Bilder und sonstiges Drittmaterial unterliegen ebenfalls der genannten Creative Commons Lizenz, sofern sich aus der Abbildungslegende nichts anderes ergibt. Sofern das betreffende Material nicht unter der genannten Creative Commons Lizenz steht und die betreffende Handlung nicht nach gesetzlichen Vorschriften erlaubt ist, ist für die oben aufgeführten Weiterverwendungen des Materials die Einwilligung des jeweiligen Rechteinhabers einzuholen.

Weitere Details zur Lizenz entnehmen Sie bitte der Lizenzinformation auf http://creativecommons.org/licenses/by/4.0/deed.de.

\section{Literatur}

Anonym (2017) https://normativ.kontur.ru/document?moduleId=1\& documentId=279426. Zugegriffen: August 2018

Anonym (2018a) Committee of the Statistics of the Kasachstan Ministry of National Economy, Tab. 16, Seite 20, unter: http://stat.gov. kz. Zugegriffen: 30. September 2018

Anonym (2018b) The Agriculture of Azerbaijan. Statistical Yearbook 2018. Seite 36; unter http://www.stat.gov.az/source/agriculture/. Zugegriffen: Aufgrufen 5. Oktober 2018

Blanke MM (2014) Äpfel ins Weltall. Obstbau 48(2):106-109

Blanke M M (2020) Obstbau in Serbien. Besseres Obst 65(8):16-18

Blanke M, Yuri A (2020) Obstbau in Chile - Exportrekorde vom Fusse der Anden. Erwerbs-Obstbau 62(2):175-180. https://doi.org/ 10.1007/s10341-020-00474-1

BMEL (2014) Der Gartenbau in Deutschland - Daten und Fakten März 2014. BLE, Bonn, 78 Seiten

FAO (2018) FAO weltweite Apfelanbaustatistiken. http://www.fao.org/ faostat/en/\#data/QC. Zugegriffen: 10. Oktober 2018

FNC (2017) Homepage des Michurin Federal Research Centre (FNC). http://fnc-mich.ru. Zugegriffen: 10. Oktober 2018

Garming H, Dirksmeyer W, Bork L (2018) Entwicklungen des Obstbaus in Deutschland von 2005 bis 2017: Obstarten, Anbauregionen, Betriebsstrukturen und Handel. JKI-Thünen-Working Paper Nr. 100. https://www.thuenen.de/de/infothek/publikationen/ thuenen-working-paper/. Zugegriffen: 20. Okotber 2018

Güll R (2015) Streuobstwiesen. Statistisches Monatsheft Baden-Württemberg 12/2015. https://www.statistik-bw.de/Service/Veroeff/ Monatshefte/20151207. Zugegriffen: 15. Oktober 2018

Rosstat (2017) Homepage des Federal State Statistics Services. http:// www.gks.ru/wps/wcm/connect/rosstat_main/rosstat/en/figures/ agriculture/. Zugegriffen: 28. September 2018 